\title{
NON-STEADY-STATE TRANSPORT OF SUPERTHERMAL ELECTRONS IN THE PLASMASPHERE
}

\author{
George V. Khazanov, Michael W. Liemohn, Tamas I. Gombosi and Andrew F. Nagy
}

Space Physics Research Laboratory, Department of Atmospheric, Oceanic and Space Science, University of Michigan

Abstract. Numerical solutions to the time-dependent kinetic equation, which describes the transport of superthermal electrons in the plasmasphere between the two conjugate ionospheres, are presented. The model calculates the distribution function as a function of time, field-aligned distance, energy, and pitch-angle. The processes of refilling, depleting, and establishing steady-state conditions of superthermal electrons in the plasmasphere are discussed.

\section{Introduction}

A distinctive feature of the ionospheric plasma is the presence of superthermal electrons. Superthermal electrons, which are created by ionization of the atmosphere by solar radiation or energetic particles of magnetospheric origin, take an active part in a number of physical processes affecting the Earth's ionosphere and plasmasphere. Knowledge of the distribution function of superthermal electrons is required when solving such geophysical problems as heating of the thermal ionospheric and plasmaspheric plasmas, optical emissions and ionization of the upper atmosphere, and wave generation in the ionospheric and plasmaspheric plasmas.

One important aspect of the superthermal electron problem is their transport through the plasmasphere. The superthermal electrons escaping from the ionosphere experience small-angle scattering when traveling through the plasmasphere as a result of the Coulomb interaction with the thermal plasma. Due to this scattering, some of the superthermal electrons are scattered to the outside of the loss cone and undergo magnetic reflection, i.e. they become trapped. Sanatani and Hanson [1970] and Nagy and Banks [1970] presented qualitative discussions of electron trapping and the resulting increase in plasmaspheric heating, but the first attempts of quantitative calculations were those of Gastman [1973], Takahashi [1973], Swartz et al. [1975], Lejeune and Worsmer [1976] and Khazanov et. al. [1977].

Among the numerous papers devoted to the superthermal electron transport between the magnetoconjugate regions of the ionosphere, no results have yet been published which describe the evolution and formation of superthermal electron fluxes under nonstationary conditions. Such models may be needed when the local source of superthermal electrons increases or decreases rather sharply, such as during sunrise and sunset, auroral precipitation events, or plasmaspheric refilling. During these short periods, strongly anisotropic distributions of superthermal electrons are generated, which may be sources of plasma instabilities and anomalous heating of the thermal plasma.

Copyright 1993 by the American Geophysical Union.

Paper number 93GL03121

0094-8534/93/93GL-03121\$03.00
Gefan and Khazanov [1990] have demonstrated the ability to model the time-dependent behavior of superthermal electrons. They simplified the problem by bounce-averaging the trapped population, and the time constants for filling the trapped region were found. The time to reach steady-state for filling a flux-tube with superthermal electrons was found to be shorter than, or of the order of, the time constant of the source for sunrise conditions. However, it was also pointed out that for the case when the flux-tubes are depleted of thermal plasma, as after a magnetic storm, the time constants associated with the refilling may be considerably longer, thus meriting a time-dependent description of the problem.

In this paper the first results of a numerical study of the non-steady-state kinetic equation for superthermal electrons in the plasmasphere are presented between the conjugate regions of the ionosphere. The distribution function in time, distance along arbitrary geomagnetic field lines, energy, and pitch-angle are among the parameters calculated by the model.

\section{Model}

The two main processes controlling the behavior of superthermal electrons in the plasmasphere are their motion in the inhomogeneous geomagnetic field and Coulomb collisions with the background thermal plasma. The kinetic equation for superthermal electrons in the guiding center approximation at altitudes greater than about $1000 \mathrm{~km}$ can be written as [Khazanov et al., 1977; 1992]:

$$
\begin{aligned}
& \frac{\beta}{\sqrt{E}} \frac{\partial \phi}{\partial t}+\mu \frac{\partial \phi}{\partial s}-\frac{1-\mu^{2}}{2} \frac{1}{B} \frac{\partial B}{\partial s} \frac{\partial \phi}{\partial \mu}= \\
& A n_{e}\left\{\frac{\partial}{\partial E}\left(\frac{\phi}{E}\right)+\frac{1}{2 E^{2}} \frac{\partial}{\partial \mu}\left[\left(1-\mu^{2}\right) \frac{\partial \phi}{\partial \mu}\right]\right\},
\end{aligned}
$$

where $\{t, s, \mu, E\}$ are time, distance along a given field line, the cosine of the pitch-angle, and energy, respectively; $\beta=1.7 \times 10^{-8} \mathrm{eV}^{1 / 2} \mathrm{~cm}^{-1} \mathrm{~s}$ is a conversion constant; $\phi$ is the flux of the electrons; $A=2 \pi \mathrm{e}^{4} \ln \Lambda=2.6 \times 10^{-12} \mathrm{~cm}^{2} \mathrm{eV}^{2}$ (e is the electron charge, $\ln \Lambda$ is the Coulomb logarithm); $\mathrm{B}(\mathrm{s})$ is the magnetic field intensity; and $n_{e}$ is the density of the thermal plasma.

In order to decrease undesirable computational effects associated with approximation errors of the derivatives $\partial / \partial s$ and $\partial / \partial \mu$, it is convenient to change variables from $(\mu, s)$ to $\left(\mu_{0}, \mathrm{~s}\right)$ [Khazanov et al., 1979], where

$$
\mu_{0}=\frac{\mu}{|\mu|} \sqrt{1-\frac{B_{0}}{B(s)}\left(1-\mu^{2}\right)}
$$

with $\mathrm{B}_{0}$ and $\mu_{0}$ denoting the magnetic field and the cosine of the pitch-angle at the magnetic equator of the flux-tube. This 


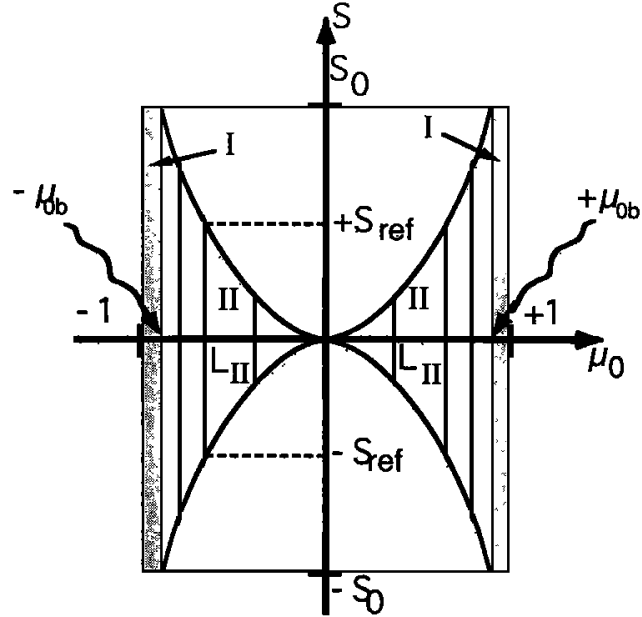

Fig. 1: Illustration of precipitation "fly-through" (I) and trapped or capture (II) zones in the $s-\mu_{0}$ plane.

change of variables is desirable because $\phi\left(\mu_{0}, s\right)$ now is a slowly varying function with $s$ ( $\mu_{0}$ is the adiabatic invariant). In this case, equation (1) becomes

$$
\begin{aligned}
& \frac{\beta}{\sqrt{E}} \frac{\partial \phi}{\partial t}+\mu \frac{\partial \phi}{\partial s}=A n_{e}\left(\frac { 1 } { 2 E ^ { 2 } } \left[\frac{\left(1-\mu^{2}\right)\left(B_{0} / B-1+\mu_{0}^{2}\right)}{\mu_{0}^{2}} \frac{\partial^{2} \phi}{\partial \mu_{0}^{2}}+\right.\right. \\
& \left.\left.\frac{\mu_{0}^{2}-2 \mu_{0}^{4}+1-\left(\mu_{0}^{2}+1\right) B_{0} / B}{\mu_{0}} \frac{\partial \phi}{\partial \mu_{0}}\right]+\frac{\partial}{\partial E}\left(\frac{\phi}{E}\right)\right\} .
\end{aligned}
$$

The region over which $\phi\left(\mu_{0}, s\right)$ is defined in terms of $\mathrm{s}$ and $\mu_{0}$ is shown in Figure 1 . The loss cone is defined by $\mu_{o_{b}} \leq\left|\mu_{0}\right| \leq 1$ and the trapping region by $\sqrt{1-B_{0} / B(s)} \leq\left|\mu_{o}\right| \leq \mu_{o_{b}}$, where $\mu_{o_{b}}=\sqrt{1-B_{0} / B\left(s_{0}\right)}$ is the loss cone boundary and $B\left(s_{0}\right)$ is the magnetic field at the ionospheric boundary. The reflection point is determined from the condition $\mu=0: B\left(s_{\text {ref }}\right)=B_{0} /\left(1-\mu_{0}^{2}\right)$.

Using the numerical technique which was developed by Khazanov et al. [1979], equation (3) is solved under the initial and boundary conditions:

$$
\begin{gathered}
\phi\left(t=0, s, \mu_{0}, E\right)=\Psi_{0}\left(s, \mu_{0}, E\right) \\
\phi\left(t, s=-s_{0}, \mu_{0}, E\right)=\Psi^{-}\left(t, \mu_{0}, E\right) ;-1 \leq \mu_{0} \leq-\sqrt{1-B_{0} / B\left(-s_{0}\right)} \\
\phi\left(t, s=s_{0}, \mu_{0}, E\right)=\Psi^{+}\left(t, \mu_{0}, E\right) ; \sqrt{1-B_{0} / B\left(s_{0}\right)} \leq \mu_{0} \leq 1 . \\
\left\{\begin{array}{l}
\frac{\partial \phi\left(\theta_{0}=0, \pi\right)}{\partial \theta_{0}}=0 ; \theta_{0}=\cos ^{-1} \mu_{0} ; \\
\phi^{+}\left(t, s, \mu_{0}=\mu_{0_{b}}, E\right)=\phi^{-}\left(t, s, \mu_{0}=-\mu_{0_{b}}, E\right) \\
\frac{\partial \phi^{+}}{\partial \theta_{0}}\left(t, s, \mu_{0}=\mu_{0_{b}}, E\right)=\frac{\partial \phi^{-}}{\partial \theta_{0}}\left(t, s, \mu_{0}=-\mu_{0_{b}}, E\right) \\
\phi\left(t, s, \mu_{0}, E=E_{\max }\right)=0 .
\end{array}\right.
\end{gathered}
$$

The numerical model could be characterized as a generalized non-steady-state multistream method taking into account energy degradation, pitch-angle focusing, pitch-angle diffusion, and field-aligned transport. With a finite-difference approximation of the derivatives

$$
\begin{gathered}
\frac{\partial \phi}{\partial t}=\frac{\phi-\phi^{i-\Delta t}}{\Delta t}, \\
\frac{\partial \phi}{\partial s}=\left\{\begin{array}{l}
\frac{\phi-\phi^{-s}}{\Delta s} ; \mu_{0}>0 \\
\frac{\phi-\phi^{+s}}{\Delta s} ; \mu_{0}<0
\end{array}\right\}, \\
\frac{\partial \phi}{\partial E}=\frac{\phi^{E}-\phi}{\Delta E},
\end{gathered}
$$

equation (3) is reduced to

$$
\frac{\partial^{2} \phi}{\partial \mu_{0}^{2}}+B \frac{\partial \phi}{\partial \mu_{0}}-C \phi=D \text {. }
$$

Expressions for the coefficients $B, C$, and D, which are functions of the variables $t, s, \mu_{0}$, and $E$, can be derived using formulas (3) and ( $8 \mathrm{a}, \mathrm{b}, \mathrm{c})$. More details of this derivation are found in Khazanov et al. [1979] and Gefan and Khazanov [1990]. The function $\phi^{i-\Delta t}$ is the value of $\phi$ at the previous time step; the functions $\phi^{+s}$ and $\phi^{-s}$ are the values of $\phi$ at the upper and lower boundaries; $\phi^{E}$ is the value of $\phi$ at the next higher energy step; and $\Delta t, \Delta E$, and $\Delta s$ are the step lengths in $t, E$, and $s$.

After these manipulations, equation (9) is solved in the region over which $\phi\left(\mu_{0}, s\right)$ is defined in terms of $s$ and $\mu_{0}$ (see Figure 1) by the run method [Gefan and Khazanov, 1990].

\section{Results}

The calculations were performed for a dipole magnetic field and assumed a field-aligned distribution of the thermal plasma in the form

$$
\mathrm{n}(\mathrm{s})=\mathrm{n}\left(\mathrm{s}_{\mathrm{o}}\right) \mathrm{B}(\mathrm{s}) / \mathrm{B}\left(\mathrm{s}_{\mathrm{o}}\right) \text {. }
$$

All calculations were conducted using an isotropic pitch-angle distribution and an exponential energy $\left[\exp \left(-\mathrm{E} / \mathrm{E}_{0}\right)\right]$ spectra at the lower boundary of the plasmasphere $\left(\mathrm{s}_{\mathrm{O}}=1000 \mathrm{~km}\right)$, with the flux-tube initially empty. The energy range for the calculations was always 1 to $50 \mathrm{eV}$ (with the boundary condition given by (7)), because with an exponential source function the fluxes above this range do not significantly affect the electrons with $\leq 30 \mathrm{eV}$.

The early stages of the refilling process forms a front of superthermal electrons propagating through the flux-tube, demonstrated in Figure 2 for $\mathrm{L}=3$ and symmetric conditions of illumination in the conjugate regions of the ionosphere. Only the normalized distribution in the loss-cone is shown because the number of the electrons in the trapped zone is very small. The fluxes decrease towards the edge of the loss cone, because, for a given energy, particles with higher pitch-angles move slower along the field line. It is clearly seen that during this initial period, strongly anisotropic distributions of superthermal electrons are generated, which may be sources of plasma instabilities.

The process of establishing the steady-state conditions for different energies of the superthermal electrons $(10 \mathrm{eV}$ and 30 


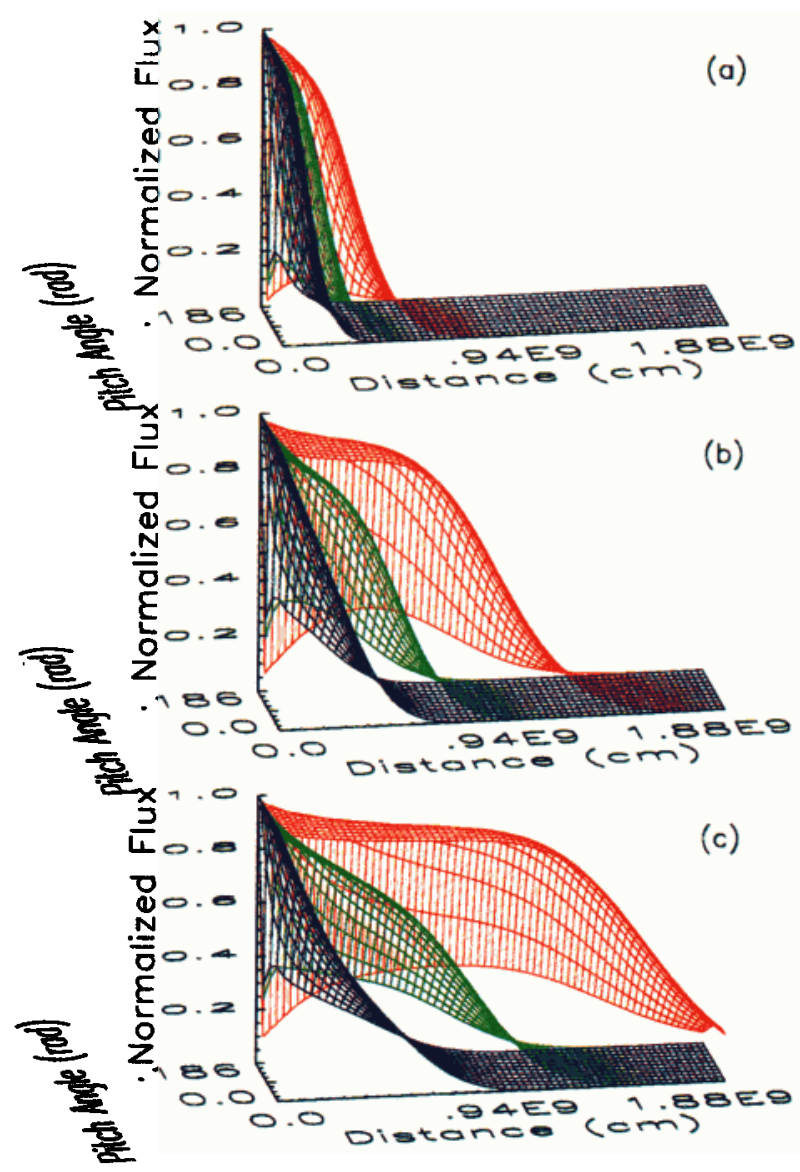

Fig. 2: Fluxes in the loss cone after 1 (a), 3 (b), and 5 (c) seconds of refilling for $\mathrm{L}=3, \mathrm{n}\left(\mathrm{s}_{\mathrm{O}}\right)=2 \times 10^{4} \mathrm{~cm}^{-3}$; the blue surfaces are $5 \mathrm{eV}$ fluxes, green surfaces are $10 \mathrm{eV}$, and red surfaces are $30 \mathrm{eV}$.

$\mathrm{eV})$ in the plasmasphere $(\mathrm{L}=3)$ is shown in Figure 3. In this case, it is convenient to normalize the flux of superthermal electrons by the steady-state flux. The loss cone reaches steady-state quickly ( $<60 \mathrm{sec}$ ) for both energies, while the trapped zone takes a much longer time. These calculations were performed for a thermal density at the lower boundary of the plasmasphere $\mathrm{n}\left(\mathrm{s}_{\mathrm{O}}\right)=5 \times 10^{2} \mathrm{~cm}^{-3}$. Such a density distribution of the thermal plasma corresponds to geomagnetically active periods in the plasmasphere. As can be seen from these calculations, by one hour the superthermal electron distribution did not reach the steady-state conditions in the trapped zone. In this case the time constants of $\leq 30 \mathrm{eV}$ superthermal electrons (which are responsible for heating the thermal plasma) become several hours, and the full timedependent electron kinetic equation must be solved for the determination of plasmaspheric heating.

Normalized equatorial steady-state pitch-angle distributions of $10 \mathrm{eV}$ electrons (illustrated in Figure 4) were calculated for the different $L$-shells with the same density $n\left(s_{0}\right)=2 \times 10^{4} \mathrm{~cm}^{-3}$ at the bottom of the plasmasphere and show how pitch-angle diffusion forms the population of the superthermal electrons in the trapped zone. The pitch-angle distribution for nonsymmetric conditions of illumination in the conjugate ionospheric regions is also shown.

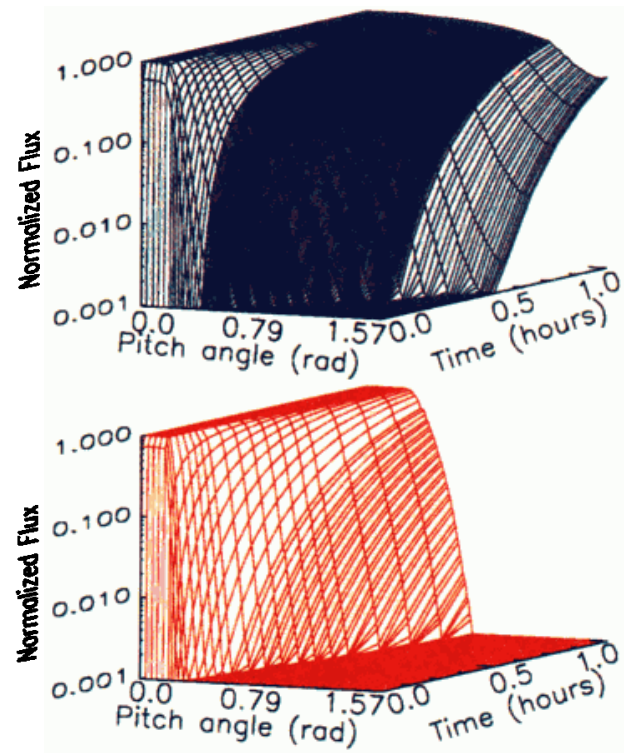

(a)

Fig. 3: Time-dependence of fluxes for different energies for $\mathrm{L}=3$ for $\mathrm{n}\left(\mathrm{s}_{\mathrm{O}}\right)=5 \times 10^{2} \mathrm{~cm}^{-3} ; 10 \mathrm{eV}(\mathrm{a})$ and $30 \mathrm{eV}(\mathrm{b})$.

Figure 5 presents the spatially normalized distribution of the omnidirectional flux after 50 seconds of plasmaspheric refilling on $\mathrm{L}=3$ depending on the average energy of superthermal electrons. It is clearly seen that the low energy population of the superthemal electrons decreases more rapidly when the flux moves to the equatorial region of the plasmasphere because the frequency of collisions with the thermal electrons increase with decreasing energy. When $E_{0}$ decreases the Coulomb energy loss increases (see eq.(1)); therefore, the amplitude of the energy spectra decreases.

And finally, Figure 6 shows the process of the depletion of the plasmasphere after turning off the ionospheric sources of superthermal electrons. Two steps were used to calculate this process. First, the steady-state solution of the kinetic equation (1) was found. Then, using this result as the initial conditions (4), and assuming zero for the boundary conditions (5) the time-dependent solution of (1) for cooling in the plasmasphere was calculated. It can be clearly seen that during plasmaspheric depletion the high energy range of the superthermal electrons takes longer to dissipate.

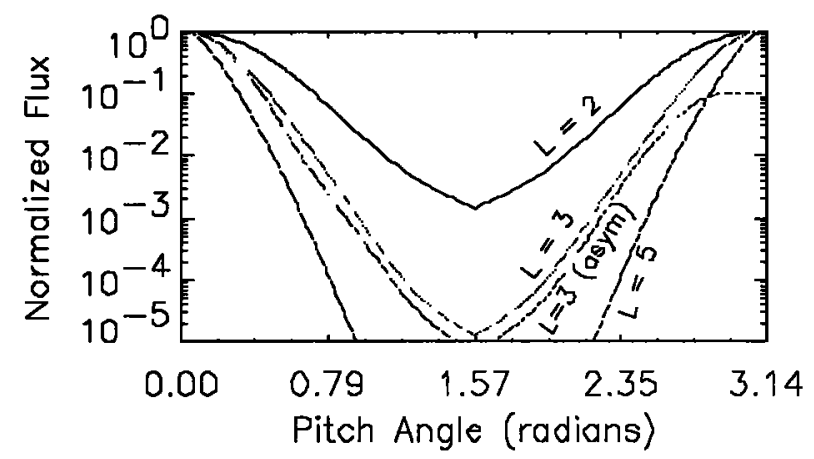

Fig. 4: Steady-state fluxes at the magnetic equator, $\mathrm{n}\left(\mathrm{s}_{\mathrm{O}}\right)=2 \times 10^{4} \mathrm{~cm}^{-3}$; for various L-shells. 


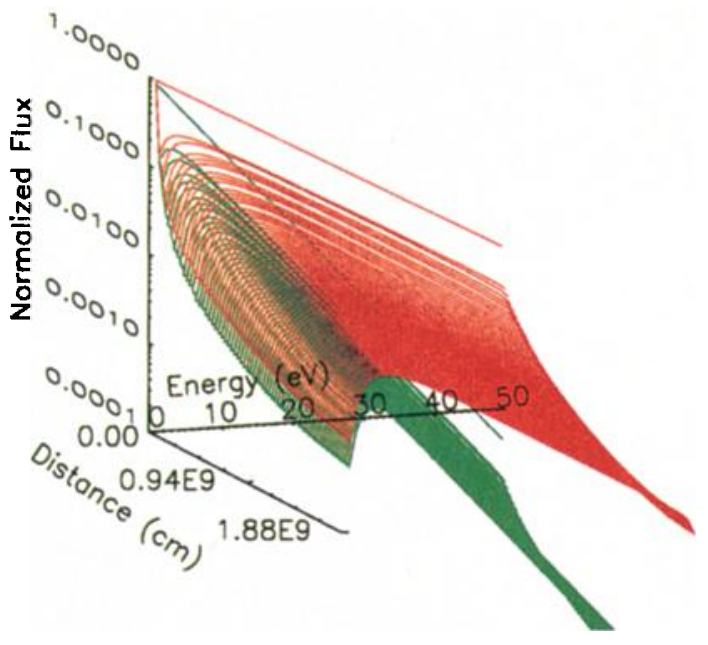

Fig. 5: Omnidirectional fluxes after 50 seconds of refilling for $\mathrm{L}=3, \mathrm{n}\left(\mathrm{s}_{\mathrm{O}}\right)=2 \times 10^{4} \mathrm{~cm}^{-3}$; the green surface is for $\mathrm{E}_{\mathrm{O}}=5 \mathrm{eV}$ and the red surface is for $E_{0}=10 \mathrm{eV}$.

\section{Summary}

The authors found the numerical solution of the nonsteady-state kinetic equation which describes the transport of superthermal electrons in the plasmasphere between the conjugate regions of the ionosphere. The distribution function in time, distance along arbitrary geomagnetic field lines, energy, and pitch-angle are among the parameters calculated by the model. Clearly, a more accurate and self-consistent set of cal-
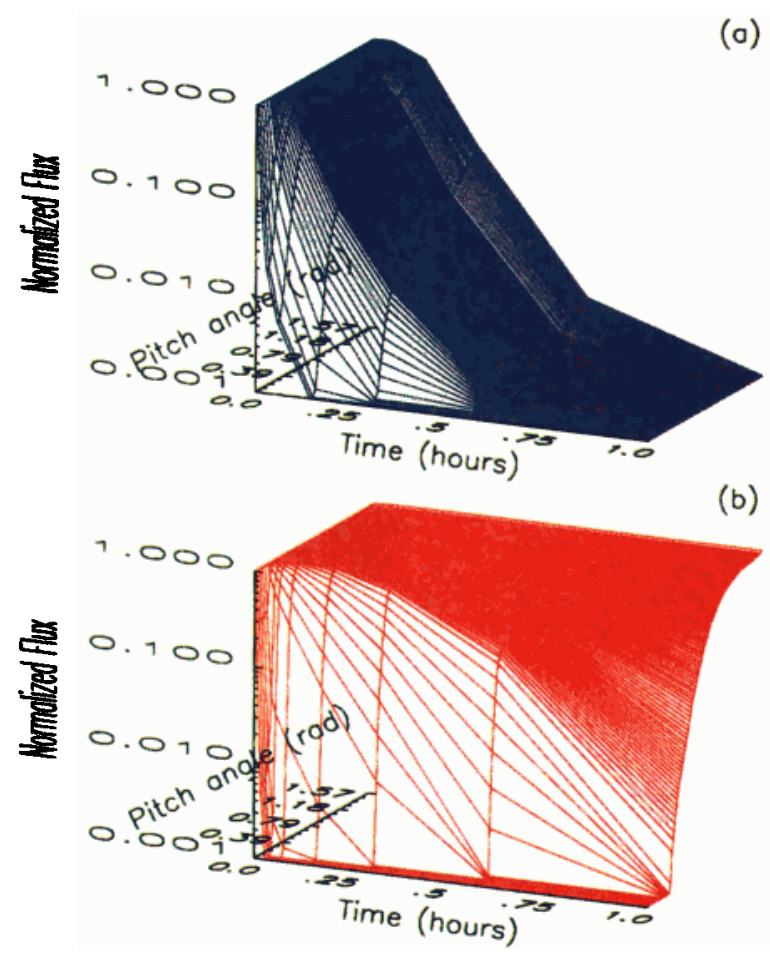

Fig. 6: Time-dependent equatorial pitch-angle distribution of fluxes for the plasmaspheric depletion for $5 \mathrm{eV}$ (a) and $20 \mathrm{eV}$ (b), $\mathrm{n}\left(\mathrm{s}_{\mathrm{o}}\right)=1 \times 10^{3} \mathrm{~cm}^{-3}$. culations would require the solutions of the electron transport equations in both the ionosphere and plasmasphere. Such calculations will be carried out in the near future. However, the use of the results presented here will, by themselves, lead to better and more quantitative model calculations.

A kinetic description of the superthermal electrons in the plasmasphere such as this will hopefully open the way for the inclusion of wave-particle interactions. It is known that plasma waves are important in the dynamics of the plasmasphere, providing a means for transfer of energy between different particle populations and diffusion of electrons across the loss cone boundary and into the upper ionosphere.

Acknowledgments. This work was supported at the University of Michigan by the National Aeronautics and Space Administration (NASA) grant NAGW-1619 and by the National Science Foundation under contract ATM-9114409.

\section{References}

Gastman, I. J., Theoretical investigation and plasma line measurements of conjugate photoelectrons in the ionosphere, Ph. D. Thesis, U. of Michigan, Ann Arbor, 1973.

Gefan, G. D., and Khazanov, G. V., Non-steady-state conditions of filling up the geomagnetic trap with superthermal electrons, Annales Geophysicae 8, 519-524, 1990.

Khazanov, G. V., M. A. Koen, and S. I. Barayschuk, Trapped photoelectrons and secondary electrons in the midlatitude plasmasphere, I, II, Cosmic research, 15, 68; 379, 1977.

Khazanov, G. V., M. A. Koen, and S. I. Burenkov. A numerical solution to the kinetic equation for photoelectrons taking into account the free and trapped zones, Cosmic research, 17, 741, 1979.

Khazanov, G. V., T. I. Gombosi, A. F. Nagy, and M. A. Koen, Analysis of the ionosphere-plasmasphere transport of superthermal electrons: 1 . Transport in the plasmasphere, $J$. Geophys. Res., 97, 16,887, 1992.

Lejeune, J. and F. Wormser, Diffusion of photoelectrons along a field line inside the plasmasphere, J. Geophys. Res., 81, 2900, 1976.

Nagy, A. F. and P. Banks, Photoelectron fluxes in the ionosphere, J. Geophys. Res., 75, 6260, 1970.

Sanatani, S. and W. B. Hanson, Plasma temperature in the magnetosphere, J. Geophys. Res., 75, 769, 1970.

Swartz, W. E., G. J. Bailey, and R. J. Moffett, Electron heating resulting from interhemispherical transport of photoelectrons, Planet. Space Sci., 23, 589, 1975.

Takahashi, T., Energy degradation and transport of photoelectrons escaping from the upper ionosphere. Rept. Ionos. and Space Res. Jap., 27, No.1, 79, 1973.

G. V. Khazanov, M. W. Liemohn, T. I. Gombosi, and A. F. Nagy, Department of Atmospheric, Oceanic, and Space Sciences, Space Physics Research Laboratory, University of Michigan, Ann Arbor, MI 48109-2143.

(Received September 24, 1993; accepted October 25, 1993.) 